\title{
Charged Higgs boson searches with the ATLAS detector
}

\author{
Elin Bergeaas Kuutmann* ${ }^{\dagger}$ \\ Uppsala University \\ E-mail: elin.bergeaas.kuutmannephysics.uu.se
}

\begin{abstract}
Many theories beyond the Standard Model, for example the Two Higgs Doublet Model (2HDM), most variants of Supersymmetry (SUSY) and the left-right symmetric model (LRSM), predict the existence of high mass charged Higgs bosons. This document presents three recent searches for charged Higgs bosons by the ATLAS experiment using $\sqrt{s}=13 \mathrm{TeV}$ of $p p$ collisions from the LHC. The analyses presented are a search for Drell-Yan pair production of doubly charged Higgs bosons decaying into same-sign charged electrons or muons using $36.1 \mathrm{fb}^{-1}$ of LHC data, and searches for singly charged Higgs bosons produced in association with a top quark and decaying either into a tau lepton and a neutrino or into a top and a bottom quark, using $13-15 \mathrm{fb}^{-1}$ of LHC data. No evidence for the existence of charged Higgs bosons were found in these studies and upper cross section limits for the production of these particles, as well as mass limits in certain benchmark scenarios are presented.
\end{abstract}

The European Physical Society Conference on High Energy Physics

5-12 July

Venice, Italy

*Speaker.

${ }^{\dagger}$ on behalf of the ATLAS collaboration. 


\section{Introduction}

Almost all extensions to the Standard Model (SM) contain an extended Higgs sector, e.g. Supersymmetry (SUSY). The simplest extension which contains a charged Higgs boson is the Two Higgs Doublet Model (2HDM) [1], which contains a total of 5 Higgs bosons: the $C P$-even neutral ones $h, H$, the $C P$-odd neutral $A$, and the charged bosons $H^{+}, H^{-}$. The free parameters of the model are $\tan \beta$ (ratio between the vacuum expectation values of the doublets), $\alpha$ (the mixing between $h$ and $H$ ) and by convention the mass of the charged Higgs boson, $m_{H^{+}}$.

There are also more elaborate extensions to the SM. By adding a Higgs triplet to SM, a total of 10 Higgs bosons emerge, four of them charged, $H^{+}, H^{-}, H^{++}, H^{--}$. Such an extension could remedy the left-right asymmetry of the weak interactions in the SM [2, 3].

This contribution to the proceedings covers recent searches by the ATLAS experiment [4] for two different models: pair-production of doubly-charged Higgs bosons decaying into charged leptons [5], $H^{++} H^{--} \rightarrow \ell^{+} \ell^{+} \ell^{-} \ell^{-}$(Sec. 2) and the production of singly charged Higgs bosons in association with top quarks [6,7], decaying into either $\tau v$ or $t b$ (Sec. 3). Figure 1 shows Feynman diagrams of these processes.
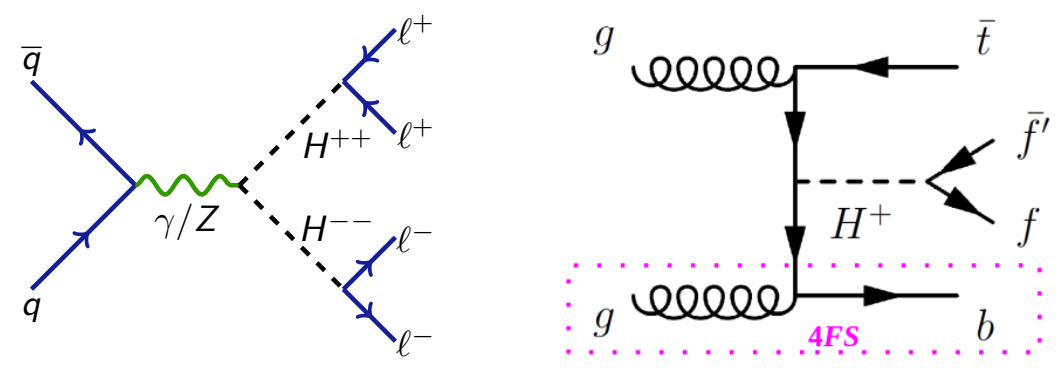

Figure 1: Feynman diagrams of the charged Higgs productions and decays considered. (Left) The pair production $q \bar{q} \rightarrow H^{ \pm \pm} H^{\mp \mp}$ process and its subsequent decay into leptons $(\ell=e$ or $\mu$ ). (Right) The associated production of $t, b$ and $H^{+}, g g \rightarrow t \bar{b} H^{+}$and the decay of the charged Higgs boson into fermions, indicating the alternative four-flavour scheme (4FS) production when the $b$-quark comes from gluon splitting.

\section{2. $H^{++} H^{--} \rightarrow \ell^{+} \ell^{+} \ell^{-} \ell^{-}$}

The ATLAS collaboration has searched for the Drell-Yan production of a $\mathrm{H}^{++} \mathrm{H}^{--}$pair, using $36.1 \mathrm{fb}^{-1}$ of LHC data collected at $\sqrt{s}=13 \mathrm{TeV}$ [5]. The analysis considered a left-right symmetric model (LRSM) [2,3]. The doubly charged Higgs bosons of this model are $H_{L}^{ \pm \pm}$and $H_{R}^{ \pm \pm}$depending on whether they couple to left- or right-handed leptons. Since electric charge must be conserved in the decay, the only allowed decay channels with SM particles are $H^{ \pm \pm} \rightarrow \ell^{ \pm} \ell^{ \pm}$or $H^{ \pm \pm} \rightarrow W^{ \pm} W^{ \pm}$. The branching ratio $(\mathrm{Br})$ of the decay depends on the Higgs boson mass $m_{H^{ \pm \pm}}$ and $v_{\Delta}$ (vacuum expectation value of the neutral Higgs triplet). For low $m_{H^{ \pm \pm}}$and low $v_{\Delta}$ the decay $H^{ \pm \pm} \rightarrow \ell^{ \pm} \ell^{ \pm}$dominates. In this analysis, the masses studied were $250 \leq m_{H^{ \pm \pm}} \leq 1300 \mathrm{GeV}$, and $v_{\Delta}=0$. Only decays into light charged same-sign leptons, $H^{++} H^{--} \rightarrow \ell^{+} \ell^{+} \ell^{-} \ell^{-}, \ell=e$ or $\mu$, were considered. There is no enhanced decay into $\tau$ leptons, since the doubly charged Higgs bosons do not couple to the lepton mass, unlike the SM Higgs boson [5]. 
The signal samples used for the analysis were Drell-Yan production of $\mathrm{H}^{++} \mathrm{H}^{--}$, simulated with Pythia8.186 [8]. The backgrounds contained either prompt leptons from $Z+V$ or $t \bar{t}+V$ (where $V=Z, W$ ); prompt leptons with a misidentified lepton charge such as $Z, t \bar{t}, W W, \ldots$; or "fakes", which contain non-prompt or mis-reconstructed leptons (e.g. multijets), estimated with the help of the data-driven "fake factor" method [9].

The selection was 2, 3 or 4 charged leptons ( $e$ or $\mu$ ), and a $b$-jet veto. Several analysis regions were defined: signal regions where most of the signal was expected, with at least one same-sign pair $\left(e^{ \pm} e^{ \pm}, e^{ \pm} \mu^{ \pm}\right.$, or $\left.\mu^{ \pm} \mu^{ \pm}\right)$, with a total of 2,3 or 4 charged leptons; control and validation regions to constrain the free fit parameters and compare the background model with data. Figure 2 (left) shows the background estimations and the data in one representative signal region with two electrons, for the invariant mass of the same-sign electron pair, $m_{e^{ \pm} e^{ \pm}}$.

Many sources of systematic uncertainties were considered. The dominant one was the uncertainty related to the fake factor method. In the $4 \ell$ channel, the statistical uncertainty was dominant, since only one single data event was found.

A maximum-likelihood fit to a mass discriminant was performed. The discriminant was the invariant mass of the same-sign lepton pair, $m\left(\ell^{ \pm} \ell^{ \pm}\right)$for the signal regions with two or three leptons, or the average invariant mass of the same-sign lepton pairs, $M=0.5 \times\left(\mathrm{m}^{++}+m^{--}\right)$, for the four-lepton signal region.

No significant excess of data above the expected SM background was observed, and $95 \%$ CL upper cross section limits with the CLs method [10] were set assuming various $\mathrm{Br}$ into $e^{ \pm} e^{ \pm}$, $e^{ \pm} \mu^{ \pm}, \mu^{ \pm} \mu^{ \pm}$or $X$, where $X$ is anything not accessible by the analysis, and couplings to left- or right-handed leptons, $H_{L}^{ \pm \pm}$or $H_{R}^{ \pm \pm}$. Figure 2 (right) illustrates the mass range excluded by the
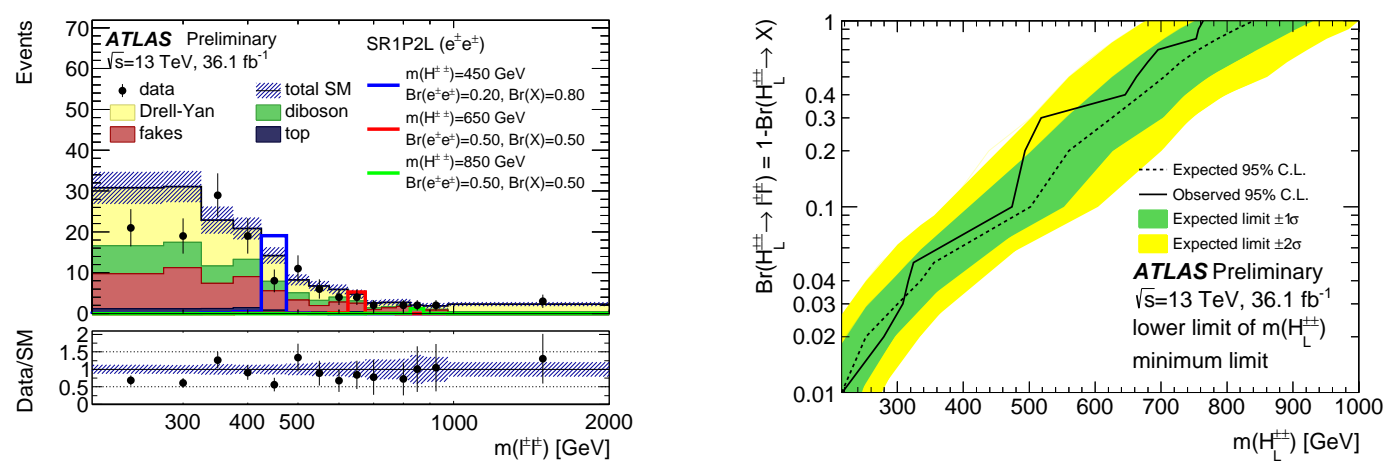

Figure 2: (Left) Distribution of $m_{\ell^{ \pm} \ell^{ \pm}}$in a representative signal region with one same-sign electronelectron pair, SR 1P2L $\left(e^{ \pm} e^{ \pm}\right)$. Solid blue, red or green lines correspond to the $H^{++} H^{--}$signal at various masses with the indicated $\mathrm{Br}$, normalised to the theoretical cross-section. The uncertainty bands include all systematic uncertainties. (Right) Limits for the Drell-Yan production of doubly charged Higgs bosons and their decay into same-sign charged lepton pairs. The figure shows lower mass limits depending on $\mathrm{Br}$ into leptons as a function of $H^{ \pm \pm}$mass. The lowest mass limit for all combinations of $B r\left(\ell^{ \pm} \ell^{ \pm}\right)=B r\left(e^{ \pm} e^{ \pm}\right)+B r\left(e^{ \pm} \mu^{ \pm}\right)+B r\left(\mu^{ \pm} \mu^{ \pm}\right)$for each fixed $B r\left(\ell^{ \pm} \ell^{ \pm}\right)$value is given. $X$ is any final state not accessible by the analysis. Figures are taken from Ref. [5].

analysis. For the assumption $\operatorname{Br}\left(e^{ \pm} e^{ \pm}\right)+\operatorname{Br}\left(e^{ \pm} \mu^{ \pm}\right)+\operatorname{Br}\left(\mu^{ \pm} \mu^{ \pm}\right)=100 \%$, the lower mass limit was found to be $m_{H_{L}}^{ \pm \pm}>770-870 \mathrm{GeV}$, where the range depends on the $\mathrm{Br}$ composition. 


\section{3. $H^{+} \rightarrow \tau v$ and $H^{+} \rightarrow t b$}

The ATLAS experiment has searched for singly charged Higgs bosons $H^{ \pm}$using between 13 and $15 \mathrm{fb}^{-1}$ of LHC data collected at $\sqrt{s}=13 \mathrm{TeV}[6,7]$.

The dominant production of singly charged Higgs bosons in most models happens in association with top quarks, as illustrated in Fig. 1 (right). In the following studies, the signal was modelled with MADGRAPH5 [11] using a 4FS approach [12]. The cross sections were combined for both 5FS (when the $b$-quark can be a sea quark in the proton) and 4FS (gluon-gluon production only) with Santander matching [13]. Only $m_{H^{ \pm}}$values above the top quark mass were considered in these studies. For the benchmark models considered, $H^{ \pm} \rightarrow t b$ or $H^{ \pm} \rightarrow \tau v$ are the dominant decay modes ${ }^{1}$. The $H^{+} \rightarrow \tau \nu$ final state is important especially at high $\tan \beta$, while $H^{+} \rightarrow t b$ is the most accessible final state at low $\tan \beta$.

In the $\tau \nu$ final state analysis, the masses considered were $200 \leq m_{H^{+}} \leq 2000 \mathrm{GeV}$. The signal selection required exactly one hadronically decaying $\tau$ lepton, while vetoing $e$ and $\mu$. The neutrino gives rise to missing transverse momentum, $E_{\mathrm{T}}^{\text {miss }}$, which was also used for triggering the events. At least three jets were required, of which at least one was $b$-tagged ${ }^{2}$. The SM backgrounds can either contain prompt $\tau$ leptons from processes such as $t \bar{t}, W / Z+$ jets, $Z, W W, W Z, Z Z$, or non-prompt $\tau$ leptons from mis-identified jets or mis-identified $e$ or $\mu$.
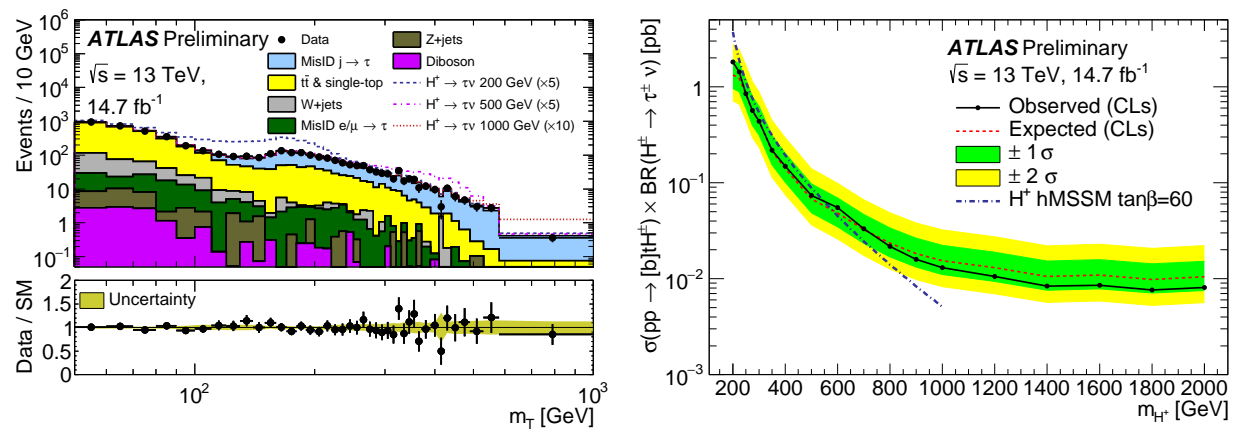

Figure 3: (Left) Expected SM background and data, in the discriminating variable $m_{\mathrm{T}}$. (Right) Modelindependent upper cross section limits as a function of the $H^{+}$mass. Figures are taken from Ref. [6].

Many sources of systematic uncertainties were considered, where the $t \bar{t}$ modelling was dominant at low $m_{H^{+}}$, and the $\tau$ leptons mis-identification estimation was dominant at high $m_{H^{+}}$. A profile likelihood fit to the discriminating variable $m_{\mathrm{T}}=\sqrt{2 p_{\mathrm{T}} E_{\mathrm{T}}^{\text {miss }}\left(1-\cos \Delta \phi_{\tau, E_{\mathrm{T}}^{\text {miss }}}\right)}$ was done. No deviation from the expected SM background was found, and upper 95\% CL limits with the CLs method were set on the cross section. Figure 3 shows the expected background composition and the data for the $m_{\mathrm{T}}$ variable, and the upper cross section limits of the analysis.

\footnotetext{
${ }^{1}$ Since neither of the discussed analyses makes any distinction between the charges of the final state particles, a short-hand notation is used: $H^{+} \rightarrow t b$ is taken to mean both $H^{+} \rightarrow t \bar{b}$ and its conjugate $H^{-} \rightarrow \bar{t} b$, while $H^{+} \rightarrow \tau \nu$ means both $H^{+} \rightarrow \tau^{+} v_{\tau}$ and $H^{-} \rightarrow \tau^{-} \bar{v}_{\tau}$

${ }^{2} \mathrm{~A}$ jet which originates from the hadronisation of a $b$-quark can be identified as such by the procedure called $b$ tagging [14], using multivariate techniques. The $b$-tagged jets used here are tagged at the $70 \%$ efficiency working point.
} 
For the $H^{+} \rightarrow t b$ decay, the $\ell+$ jets final state was considered, when one of the top quarks in $t b H^{+} \rightarrow t b t b$ decays semi-leptonically. In the analysis, exactly one $e$ or $\mu$ was selected in events with least 4 jets of which at least 2 were $b$-tagged. The most challenging SM background was the irreducible $t \bar{t}+b \bar{b}$. Several signal and control regions (SR, CR) were defined based on jet and $b$-jet multiplicity, counting 4, 5 or 6 or more jets and 2, 3 and 4 or more $b$-tagged jets.

To suppress the $t \bar{t}$ background, Boosted Decision Trees (BDT) were trained for each mass point and SR. The background in the training was $t \bar{t}+\geq 1 b$ for $m_{H^{+}} \leq 500 \mathrm{GeV}$, and all $t \bar{t}$ backgrounds for $m_{H^{+}}>500 \mathrm{GeV}$. The analysis was done through a simultaneous profile likelihood fit in all SR and CR. The fitted variable was the BDT output in the SR, and $H_{\mathrm{T}}^{\text {had }}$ (the scalar sum of the $p_{\mathrm{T}}$ of all jets) in the CR. Many systematic uncertainties were considered, where the main ones originate from the modelling of $t \bar{t}$ produced in association with heavy flavour jets, $b$-tagging and jet energy scale and resolution. No significant excess of data over the expected background was found and upper 95\% CL limits on the cross section as a function of $m_{H^{+}}$were set using the CLs method. Figure 4 shows a comparison between the expected SM background and data, as well as the upper cross section limits as a function of mass.
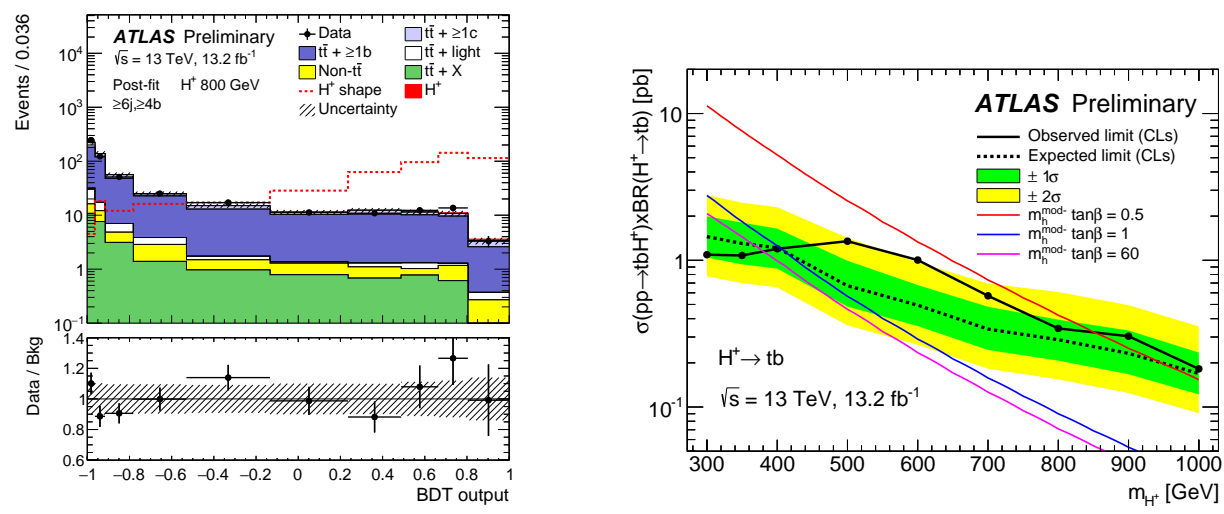

Figure 4: (Left) The output for a BDT trained at the $H^{+}$mass of $800 \mathrm{GeV}$ after a fit to data, comparing the expected SM background and data. (Right) The upper cross section limits as a function of the $H^{+}$mass, indicating theoretical cross sections for three $\tan \beta$ values in the $m_{\mathrm{h}}^{\bmod -}$ scenario $[15,16,17]$. Figures are taken from Ref. [7].

Additionally, interpretations in the $h \operatorname{MSSM}[18,19]$ and MSSM $m_{\mathrm{h}}^{\bmod -}[15,16,17]$ models were given. Many $H^{+}$mass points at high $\tan \beta$ were excluded, as indicated in Fig. 5.

\section{Summary and conclusions}

Charged Higgs bosons are needed in many BSM scenarios. The work presented in Ref. [5] and referred in this contribution improves the previous ATLAS limits on the $H^{++} H^{--} \rightarrow \ell^{+} \ell^{+} \ell^{-} \ell^{-}$ production and decay based on $\sqrt{s}=8 \mathrm{TeV}$ LHC data [9]. For the searches of singly charged Higgs bosons [6, 7], the $t b$ and $\tau v$ final states are complementary, together excluding certain BSM scenarios at very high and very low $\tan \beta$. While no signal has been found yet, there is plenty of parameter space left to explore. 

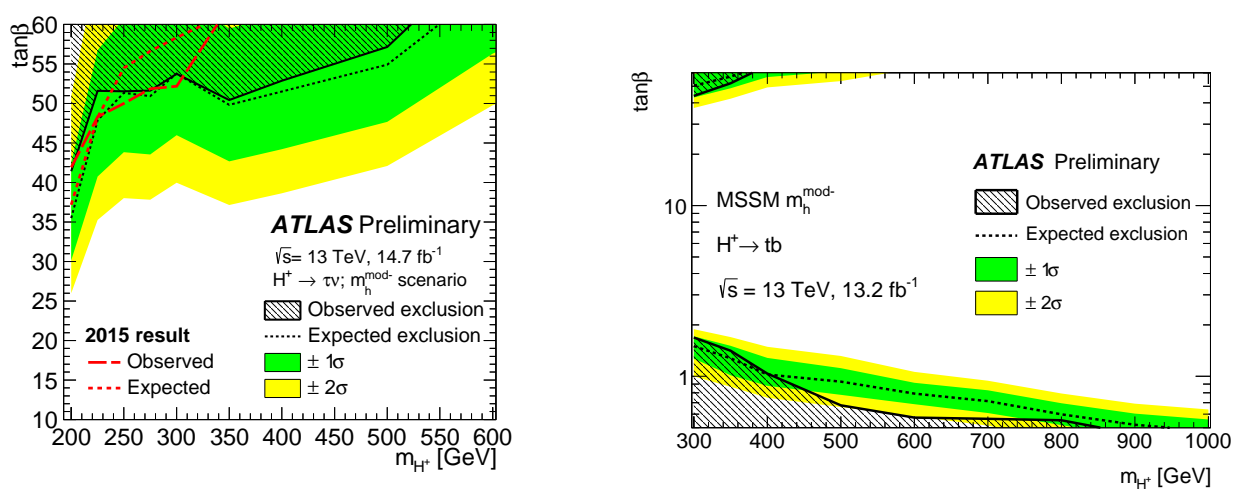

Figure 5: Model exclusion limits for the $m_{\mathrm{h}}^{\bmod -}$ scenario $[15,16,17]$. Exclusion limits for (left) $H^{+} \rightarrow \tau v$ and (right) $H^{+} \rightarrow t b$, as a function of $\tan \beta$ and the $H^{+}$mass. Figures are taken from Refs. [6, 7].

\section{References}

[1] G.C. Branco et al., Theory and phenomenology of two-Higgs-doublet models, Phys.Rept. 516, p 1-102, (2012), [arXiv:1106.0034 [hep-ph]].

[2] G. Senjanovic and R. N. Mohapatra, Exact left-right symmetry and spontaneous violation of parity, Phys. Rev. D12 (1975) 1502.

[3] G. Senjanovic, Spontaneous breakdown of parity in a class of gauge theories, Nuclear Physics B 153 Supplement C, pp 334 - 364 (1979)

[4] ATLAS Collaboration, The ATLAS Experiment at the CERN Large Hadron Collider, JINST 3 (2008) S08003.

[5] ATLAS Collaboration, Search for doubly-charged Higgs boson production in multi-lepton final states with the ATLAS detector using proton-proton collisions at $\sqrt{s}=13 \mathrm{TeV}$, ATLAS-CONF-2017-053, http://cds.cern.ch/record/2273869.

[6] ATLAS Collaboration, Search for charged Higgs bosons in the $\tau+$ jets final state using $14.7 \mathrm{fb}^{-1}$ of pp collision data recorded at $\sqrt{s}=13 \mathrm{TeV}$ with the ATLAS experiment, ATLAS-CONF-2016-088, http://cds.cern.ch/record/2206282.

[7] ATLAS Collaboration, Search for charged Higgs bosons in the $H^{ \pm} \rightarrow$ tb decay channel in $p p$ collisions at $\sqrt{s}=13 \mathrm{TeV}$ using the ATLAS detector, ATLAS-CONF-2016-089, https://cds.cern.ch/record/2206809.

[8] T. Sjöstrand, et al.,A Brief Introduction to PYTHIA 8.1, Comput. Phys. Commun. 178 p. 852 (2008), [arXiv:0710.3820 [hep-ph]].

[9] ATLAS Collaboration, Search for anomalous production of prompt same-sign lepton pairs and pair-produced doubly charged Higgs bosons with $\sqrt{s}=8 \mathrm{TeV}$ pp collisions using the ATLAS detector, JHEP 1503 (2015) 041, [arXiv:1412.0237 [hep-ex]].

[10] A. Read, Presentation of search results: the $C L_{s}$ technique, J. Phys. G28, pp. 2693-2704 (2002).

[11] J. Alwall, et al., The automated computation of tree-level and next-to-leading order differential cross sections, and their matching to parton shower simulations, JHEP 1407(2014) 079, [arXiv:1405.0301 [hep-ph]]. 
[12] C. Degrande et al., Heavy charged Higgs boson production at the LHC, JHEP 1510 (2015) 145 [arXiv:1507.02549 [hep-ph]].

[13] R. Harlander et al., Bottom-quark associated Higgs-boson production: reconciling the four-and five-flavour scheme approach, arXiv:1112.3478 [hep-ph].

[14] ATLAS Collaboration, Performance of b-Jet Identification in the ATLAS Experiment, JINST 11 (2016) no.04, P04008 [arXiv:1512.01094 [hep-ex]].

[15] S. Heinemeyer et al. [LHC Higgs Cross Section Working Group], Handbook of LHC Higgs Cross Sections: 3. Higgs Properties, arXiv:1307.1347 [hep-ph].

[16] M. Carena, et al., MSSM Higgs Boson Searches at the LHC: Benchmark Scenarios after the Discovery of a Higgs-like Particle, Eur. Phys. J. C 73 (2013) no.9, 2552 [arXiv:1302.7033 [hep-ph]].

[17] D. de Florian et al. [LHC Higgs Cross Section Working Group], Handbook of LHC Higgs Cross Sections: 4. Deciphering the Nature of the Higgs Sector, arXiv:1610.07922 [hep-ph].

[18] A. Djouadi, et al. The post-Higgs MSSM scenario: Habemus MSSM?, Eur. Phys. J. C 73 (2013) 2650 [arXiv:1307.5205 [hep-ph]].

[19] A. Djouadi et al., Fully covering the MSSM Higgs sector at the LHC, JHEP 1506 (2015) 168 [arXiv:1502.05653 [hep-ph]]. 